EDITORIAL

\title{
Drug-Induced QT Interval Prolongation in Children: Are the Kids Alright?
}

\author{
James E Tisdale
}

$\mathrm{T}$ orsades de pointes $(\mathrm{TdP})$ is a polymorphic ventricular tachycardia associated with prolongation of the heart ratecorrected QT (QTc) interval on an electrocardiogram (ECG). ${ }^{1}$ TdP can be catastrophic, as it may result in sudden cardiac death. ${ }^{1}$ QTc interval prolongation may be inherited or acquired. The inherited form is represented by congenital long QT syndrome, of which there are at least 15 types, ${ }^{2}$ whereas the acquired form is most commonly caused by drugs. ${ }^{3}$ In the past 3 decades, several drugs, including astemizole, cisapride, grepafloxacin, prenylamine, and terfenadine, have been withdrawn from the Canadian market because of deaths due to TdP. ${ }^{4}$ Nonetheless, more than 100 drugs with the potential to prolong the QTc interval and cause TdP remain available. ${ }^{5}$

Although most clinicians are familiar with the potential for drug-induced QTc interval prolongation and TdP in adults, many are less aware that this adverse drug reaction can also occur in pediatric populations. However, many cases of drug-induced $\mathrm{TdP}$ in children have been published. ${ }^{6}$ Pediatric cases of TdP have been associated with various drugs, including astemizole, cisapride, pentamidine, risperidone, antiarrhythmic agents (amiodarone, procainamide, and sotalol), and antifungals (fluconazole and voriconazole). ${ }^{6}$

In the current issue of the Canadian Journal of Hospital Pharmacy, Morris and others ${ }^{7}$ review the literature regarding QT interval prolongation associated with domperidone in nononcologic pediatric patients. Domperidone is the gastrointestinal promotility agent of choice for pediatric gastroesophageal reflux disease, which typically occurs between 5 and 18 months of age. These authors describe the findings of 3 prospective cohort studies, 1 randomized controlled trial, and 1 case report of domperidone use in a total of 137 children ranging in age from 2 days to 9 months. ${ }^{7}$ For most of these patients, QTc interval prolongation did not occur, but for 6 patients the QTc interval was abnormally long ( $\geq 450 \mathrm{~ms}$ ), including 3 with QTc interval above $460 \mathrm{~ms}$, to a maximum of $490 \mathrm{~ms}^{8}$ In one of the prospective studies, domperidone was associated with significant lengthening of the mean QTc interval relative to pretreatment values in neonates. ${ }^{9}$ Although none of the patients represented in this literature review had a QTc interval above $500 \mathrm{~ms}$ or experienced an increase in QTc interval of more than $60 \mathrm{~ms}$ relative to the pretreatment value (the generally accepted thresholds for a marked increase in risk of $\mathrm{TdP}^{6}$ ), druginduced QTc interval pro-

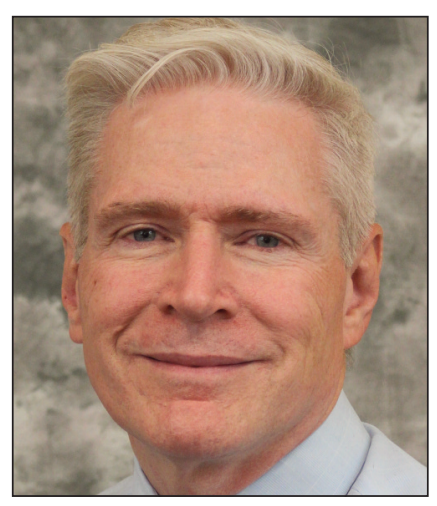
longation to greater than $450 \mathrm{~ms}$ is reason for concern. Fortunately, none of the patients in this analysis experienced TdP or sudden cardiac death.

The overall incidence of drug-induced TdP is not well described for adult populations and is generally unknown for pediatric populations. The incidence of $\mathrm{TdP}$ associated with various drugs (determined in overwhelmingly adult populations) ranges from $2 \%$ to $12 \%$, depending on the drug, the dose, and other risk factors. ${ }^{3}$ In one of the reports reviewed by Morris and others, ${ }^{7}$ the incidence of QTc interval prolongation associated with domperidone in infants was $4.4 \% .{ }^{10}$

Drug-induced QTc interval prolongation and TdP depend heavily on risk factors and occur only rarely in patients without concomitant predisposing factors. ${ }^{6}$ For adults, the risk factors include female sex; advanced age (> 65 years); hypokalemia, hypomagnesemia, or hypocalcemia; heart failure with reduced ejection fraction; bradycardia; treatment with more than one QTc interval-prolonging drug; and conditions leading to elevated plasma concentrations of such drugs, such as kidney or liver disease, drug interactions, and rapid IV administration. ${ }^{6}$ It is unknown which of these risk factors (excluding older age) also apply to pediatric populations. In one small study $(n=31)$, independent risk factors for domperidone-associated QTc interval prolongation in neonates were advanced gestational age and, paradoxically, serum potassium concentration at the upper limit 
of normal. ${ }^{9}$ The reason for an association with high-normal serum potassium concentration, rather than the widely accepted risk factor of hypokalemia, is unclear. Given what is known about effects on potassium channels, it seems likely that, similar to the situation for adults, hypokalemia, hypomagnesemia, bradycardia, and elevated plasma concentrations of QTc interval-prolonging drugs are risk factors for pediatric patients. ${ }^{6}$ However, female sex is not a risk factor for QTc interval prolongation and TdP in prepubertal children. Evidence shows that the QTc interval is similar in boys and girls until the onset of puberty, at which time QTc intervals diverge. ${ }^{11}$ This divergence is likely the result of increasing testosterone production at puberty in males, which has been shown to be associated with a shorter QTc interval. ${ }^{6}$ Therefore, female sex is a risk factor for QTc interval prolongation and TdP only at or following puberty.

Recommendations for hospitalized adult patients receiving therapy with a QTc interval-prolonging drug include continuous monitoring of the QTc interval and maintenance of serum potassium, magnesium, and calcium concentrations within the normal range. ${ }^{1}$ If the patient's QTc interval exceeds $500 \mathrm{~ms}$ or increases by more than $60 \mathrm{~ms}$ over the pretreatment value, recommended interventions include use of alternative, non-QTc interval-prolonging therapy, where possible; assessment of potential drug interactions, bradyarrhythmias, or electrolyte abnormalities; and readiness to manage $\mathrm{TdP}$ should it occur. ${ }^{1}$ Recommendations for hospitalized children or neonates receiving therapy with QTc interval-prolonging drugs have not been published, but it seems reasonable to promulgate recommendations similar to those for adults. For pediatric patients with risk factors for TdP but for whom continuous QTc interval monitoring is not feasible, a 12-lead ECG should be obtained at baseline and then 3-7 days after initiation of QTc interval-prolonging therapy.

The review by Morris and others ${ }^{7}$ serves as a reminder that drug-induced QTc interval prolongation is not restricted to adults and that children and neonates are also susceptible, particularly if they have predisposing factors. Appropriate QTc interval monitoring and attention to modifiable risk factors are important for reducing the risk of QTc interval prolongation and TdP in children and neonates receiving therapy with QTc intervalprolonging drugs.

\section{References}

1. Drew BJ, Ackerman MJ, Funk M, Gibler WB, Kligfield P, Menon V, et al.; American Heart Association Acute Cardiac Care Committee of the Council on Clinical Cardiology, the Council on Cardiovascular Nursing, and the American College of Cardiology Foundation. Prevention of torsade de pointes in hospital settings: a scientific statement from the American Heart Association and the American College of Cardiology Foundation. Circulation. 2010;121(8):1047-60. Erratum in: Circulation. 2010;122(8):e440.
2. Mizusawa Y, Horie M, Wilde AAM. Genetic and clinical advances in congenital long QT syndrome. Circ J. 2014;78(12):2827-33.

3. Tisdale JE. Drug-induced QT interval prolongation and torsades de pointes: role of the pharmacist in risk assessment, prevention and management. Can Pharm J. 2016;149(3):139-52.

4. Lexchin J. Drug withdrawals from the Canadian market for safety reasons, 1963-2004. CMAJ. 2005;172(6):765-7.

5. Woosley RL, Romero KA. QTDrugs Lists [database]. Oro Valley (AZ): AZCERT, Inc; [cited 2016 Apr 20]. Available from: https://www. crediblemeds.org/index.php/login/dlcheck

6. Tisdale JE. Ventricular arrhythmias. In: Tisdale JE, Miller DA, editors. Druginduced diseases. Prevention, detection and management. 2nd ed. Bethesda (MD): American Society of Health-System Pharmacists; 2010. p. 485-515.

7. Morris AD, Chen J, Lau E, Poh J. Domperidone-associated QT interval prolongation in non-oncologic pediatric patients: a review of the literature. Can J Hosp Pharm. 2016;69(3):224-30.

8. Günlemez A, Babaoglu A, Arisoy AE, Türker G, Gökalp AS. Effect of domperidone on the QTc interval in premature infants. J Perinatol. 2010; 30(1):50-3.

9. Djeddi D, Kongolo G, Lefaix C, Mounard J, Léké A. Effect of domperidone on QT interval in neonates. J Pediatr. 2008;153(5):663-6.

10. Vieira MC, Miyague NI, Van Steen K, Salvatore S, Vandenplas Y. Effects of domperidone on QTc interval in infants. Acta Paediatr. 2012;101(5):494-6.

11. Rautaharju PM, Zhou SH, Wong S, Calhoun HP, Berenson GS, Prineas R, et al. Sex differences in the evolution of the electrocardiographic QT interval with age. Can J Cardiol. 1992;8(7):690-5.

James E Tisdale, BScPharm, PharmD, is Professor, Department of Pharmacy Practice, College of Pharmacy, Purdue University, and Adjunct Professor, School of Medicine, Indiana University, Indianapolis, Indiana. $\mathrm{He}$ is also an Associate Editor for the Canadian Journal of Hospital Pharmacy.

Competing interests: James Tisdale is a volunteer member of the Advisory Board for the QTDrugs Lists database for the website www. crediblemeds.org (cited as ref. 5 in this editorial). No other competing interests were declared.

\section{Address correspondence to:}

Dr James E Tisdale

Department of Pharmacy Practice

College of Pharmacy

Purdue University

640 Eskenazi Avenue

Indianapolis IN 46202 USA

e-mail: jtisdale@purdue.edu 